\title{
Stability analysis of single-layer special-shaped folded-plane latticed shell
}

\author{
Jiajun $\mathrm{Li}^{1}{ }^{1, *}$, Jing Yang ${ }^{2}$, Yunan $\mathrm{Li}^{1}$, Jian Yang ${ }^{1}$ \\ ${ }^{1}$ Inner Mongolia Technical College of Mechanics and Electrics, Hohhot 010070, China \\ ${ }^{2}$ Highway Bureau of Inner Mongolia Autonomous Region, Hohhot 010020, China
}

\begin{abstract}
Single-layer special-shaped folded-plane latticed shell had been applied in Shenyang culture and art center. In order to analysis its static stability, explore the form of buckling modes and find out its critical load factor, this paper analysis the optimized structure with MIDAS Gen. Eigenvalue buckling analysis and nonlinear buckling analysis showed that the in-plane stiffness of the structure is very large, and the outplane stiffness is small, the upside structure is prone to instability. The critical load factor for this structure is 10.32 , the structural overall stability is fine according to the code. This structures is sensitive to the initial geometric imperfections which could lower structural stability of the system.
\end{abstract}

\section{Research background}

Latticed shell is a kind of spatial structure, which is widely used in large-span structure because of its beautiful shape, good stress and low cost. This structure is very sensitive to the initial imperfection, and its bearing capacity is generally controlled by the stability, so it is necessary to calculate and analyze the stability of the structure. The application of single-layer folded surface latticed shell is less, and its research is also less. Based on the existing stability analysis theory of latticed shell, this paper analyzes the overall stability of the steel structure roof of Shenyang Culture and Art Center, which is a special-shaped latticed shell. The results can be used for experimental study and comparison, and accumulate experience for this type of latticed shell research.

\section{General engineering situation}

Shenyang Culture and Art Center is located in Heping District of Shenyang. The roof of the project is a Singlelayer special-shaped folded-plane latticed shell with steel. The latticed shell is composed of many triangular meshes with disordered size and direction. There are 64 planes in the whole roof. Each plane triangle has different shapes and angles. The large triangle of each face is divided into 16 small triangles, as shown in Fig. 1. The overall dimension of the roof is $179 \mathrm{~m} \times 112 \mathrm{~m} \times 60 \mathrm{~m}$. The main structure of the roof is mainly composed of four parts: the main component, the secondary component, the support and the main connection node. The main components are mainly made of large diameter thick walled steel pipes with diameter of $\varphi 1430 \mathrm{~mm} \times 40 \mathrm{~mm}, \quad \varphi 1370 \mathrm{~mm} \times 65 \mathrm{~mm}$ and $\varphi$ $1370 \mathrm{~mm} \times 75 \mathrm{~mm}$. The secondary components are mainly made of two kinds of steel pipes, $\varphi 775 \mathrm{~mm} \times 30 \mathrm{~mm}$ and $\varphi$ $760 \mathrm{~mm} \times 25 \mathrm{~mm}$. The main components are connected by cast steel joints, and the main and secondary members are welded through each other. The whole roof weighs 11000 tons.

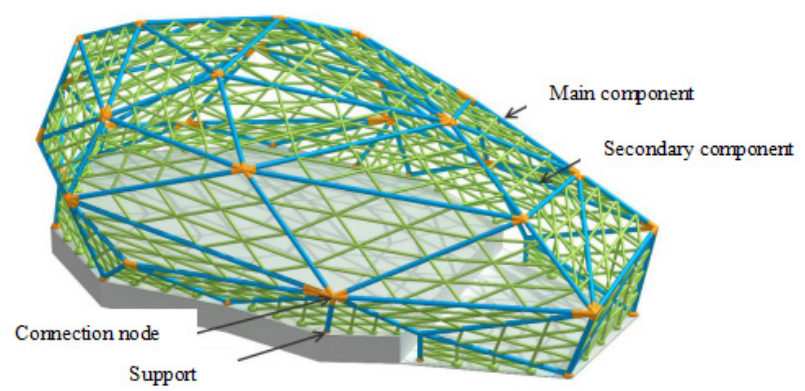

Fig. 1. Main structure of steel roof

\section{Load conditions}

\subsection{Permanent load}

Structural permanent load: because the main components are connected by cast steel joints, the weight of the joints is large, so it is calculated as 1.1 times of the permanent weight.

Roof permanent load is $1.0 \mathrm{kN} / \mathrm{m}^{2}$.

\subsection{Live load}

Roof live load is $2.0 \mathrm{kN} / \mathrm{m}^{2}$.

\footnotetext{
*Corresponding author: 83368638@qq.com
} 


\subsection{Wind load}

Basic wind pressure is $0.55 \mathrm{kN} / \mathrm{m}^{2}(\mathrm{n}=50)$. Terrain roughness class is $\mathrm{B}$.

\subsection{Snow load}

Basic snow pressure is $0.60 \mathrm{kN} / \mathrm{m}^{2}$.

\subsection{Earthquake action}

Seismic precautionary intensity class is 7. Classification of design earthquake is the first class. Design characteristics period of ground motion is $0.35 \mathrm{~s}$. Design basic acceleration of ground motion is $0.10 \mathrm{~g}$.

\subsection{Temperature load}

Temperature difference is between $-40^{\circ} \mathrm{C}$ and $40^{\circ} \mathrm{C}$.

\section{Finite element model}

The software Midas Gen is used in the finite element model analysis. The optimized model of overall structure is shown in Fig. 2. In this model, the beam element is used to simulate the members. There are 1513 elements in total. The steel material trade-mark is Q345D. There are 48 supports for the steel structure roof, all of which are calculated as fixed supports, as shown in Fig. 2.

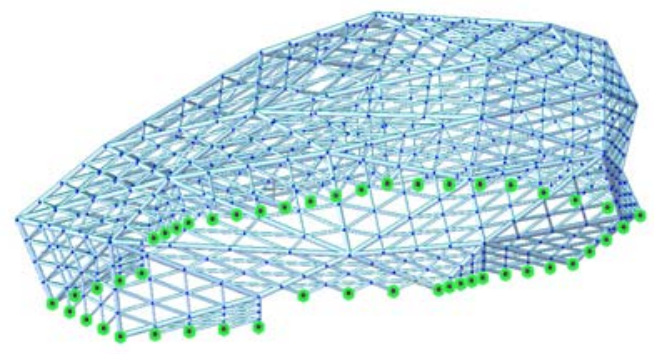

Fig. 2. Model diagram

\section{Stability analysis of shell}

\subsection{Linear buckling analysis}

Linear buckling analysis, also known as eigenvalue buckling analysis, is an ideal stability analysis method for latticed shell, that is, without considering the initial imperfections and the influence of nonlinear factors in the construction process, the calculation results are the theoretical values of buckling strength in linear elastic state.

In Midas Gen, the critical load of the structure can be calculated as follows:

$$
P_{\mathrm{cr}}=\mu P_{L L}+P_{D L}
$$

$P_{\text {cr }} \longrightarrow$ critical load of structure

$\mu \_$critical load factor (characteristic value )

$P_{L L} \longrightarrow$ variable load case
$P_{D L}$ - invariant load case

The buckling mode and the corresponding critical load factor can be obtained from (1).

Firstly, the load combination of linear buckling analysis is defined as variable load case and invariant load case, and the combination coefficient is 1.0. In the linear buckling analysis, invariant load case set as 0 , so the critical load of the structure is the product of the critical load coefficient and the variable load case. Through linear buckling analysis, the first six order eigenvalues of the shell structure are obtained, as shown in Table 1, and the first six order buckling modes are shown in Fig. 3.

Table 1. First six order eigenvalues of linear buckling analysis

\begin{tabular}{cccccccc}
\hline $\begin{array}{c}\text { Modal number } \\
\text { of linear } \\
\text { buckling } \\
\text { analysis }\end{array}$ & 1 & 2 & 3 & 4 & 5 & 6 \\
\hline Eigenvalues & 13.31 & 16.23 & 19.63 & 21.44 & 23.32 & 25.53 \\
\hline
\end{tabular}

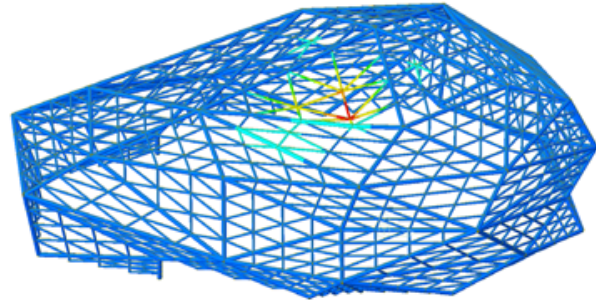

The first order buckling mode

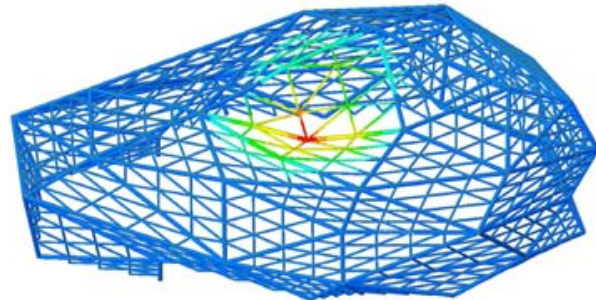

The second order buckling mode

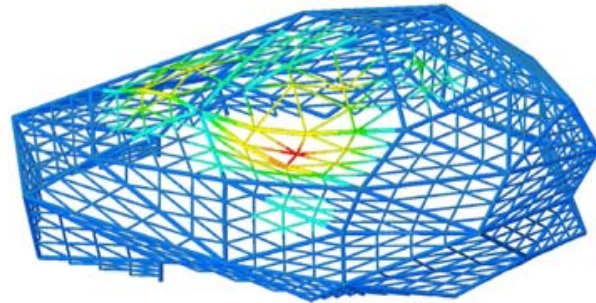

The third order buckling mode

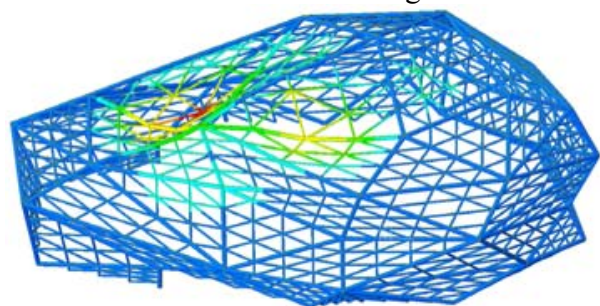

The fourth order buckling mode 


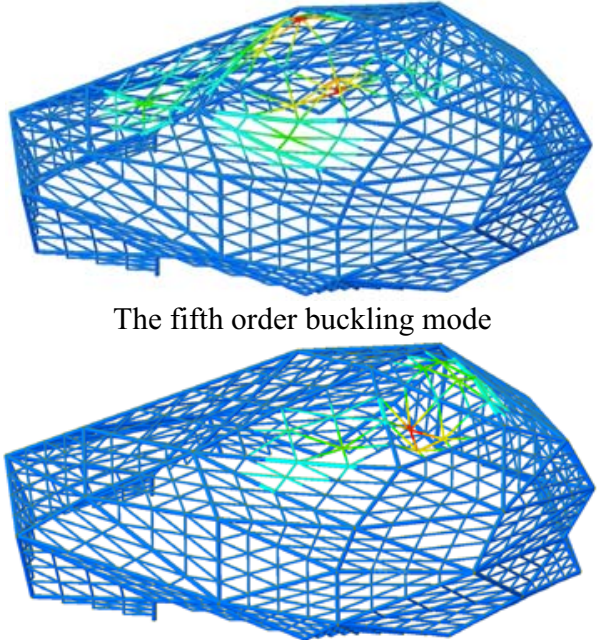

The sixth order buckling mode

Fig. 3. First six order buckling modes of linear buckling analysis

\subsection{Nonlinear buckling analysis}

As a practical project, it is not enough to do linear buckling analysis. Due to the manufacturing error, transportation loss, installation error and material defects of steel structure members, all these can not be ignored in the structural stability analysis. Therefore, it is necessary to analyze the nonlinear buckling of the structure. It is specified in the code for Technical Specification for Space Frame Structures that the stability of latticed shells can be analyzed according to geometric non-linearity. When the initial imperfections are distributed as the lowest buckling mode, that is, the first buckling mode, the calculated stability bearing capacity is the most unfavorable. At present, the main methods to consider the stability of single-layer latticed shell with initial geometric imperfections are random imperfection mode method, consistent imperfection mode method and $\mathrm{N}$-order eigenvalue imperfection mode method ${ }^{[1]}$. In this paper, the consistent imperfection mode method is used for analysis, that is, the buckling vector corresponding to the first buckling mode determined by linear buckling analysis is taken as the initial defect of the structure, and the structure is introduced for geometric nonlinear analysis.

Before nonlinear buckling analysis, it is necessary to define a load case. This is because the load effect can be superimposed in linear analysis, but not nonlinear, so the load type and coefficient to be considered must be combined into one working condition. In the analysis, the combination of 1.0SL +1.0DL + 1.0LL is taken as the load case of nonlinear analysis, where SL represents the self weight, DL represents the permanent load, and LL represents the live load. In the nonlinear buckling analysis, only geometric non-linearity is selected, and the displacement control method is selected and the control parameters are input. According to reference [2], $1 / 300(37.33 \mathrm{~mm})$ of the short span length is taken for analysis and calculation, and no defect $(0 \mathrm{~mm})$ and $1 /$ $150(74.67 \mathrm{~mm})$ are taken for comparison. No.455 node (as shown in Fig. 4) is taken as the control node to obtain the critical coefficient under different initial defects. The results are shown in Table 2 . The critical load factor displacement curve of No. 455 joint is shown in Fig. 5.

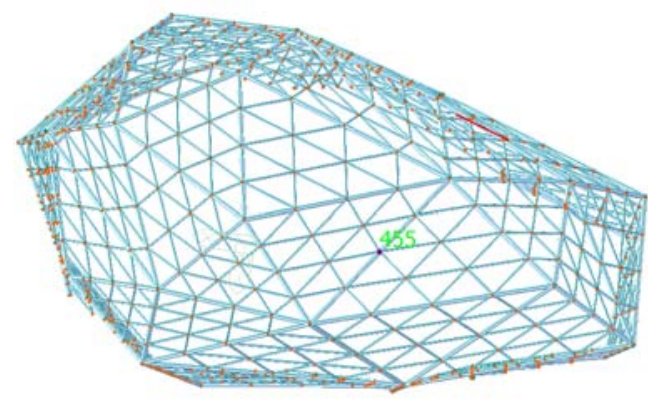

Fig. 4. Position of No.455

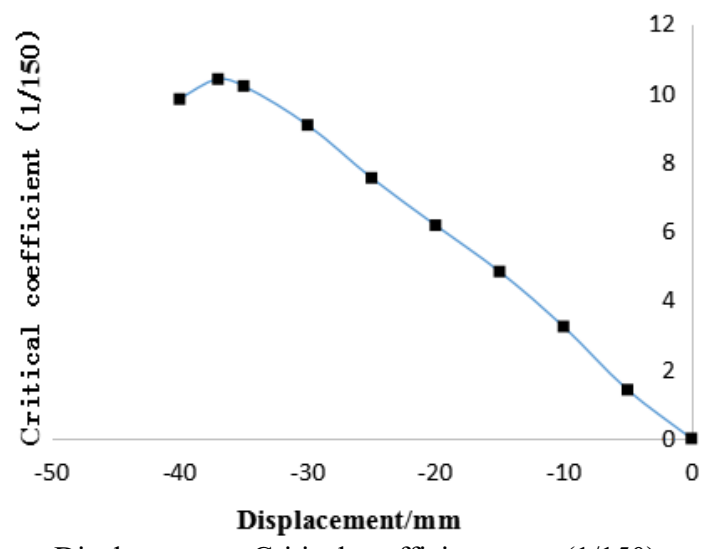

Displacement - Critical coefficient curve(1/150)

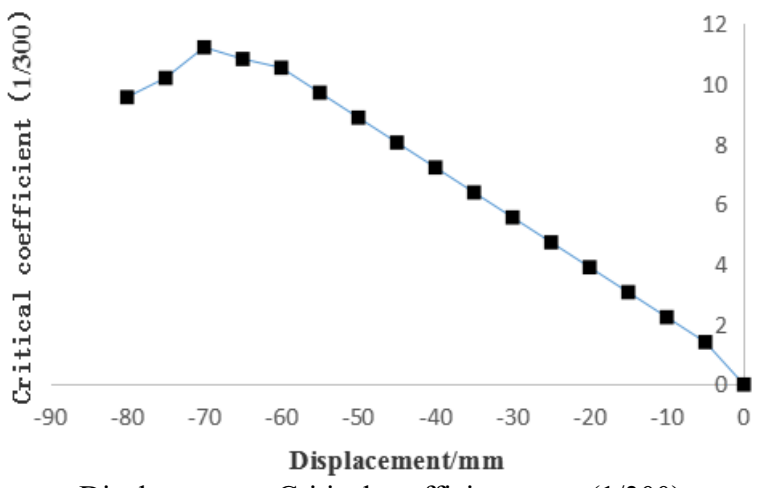

Displacement - Critical coefficient curve(1/300)

Fig. 5. Displacement - Critical coefficient curve of No.455

\section{Conclusions}

In order to describe the analysis results conveniently, the structure is divided as follows, as shown in Fig. 6. 


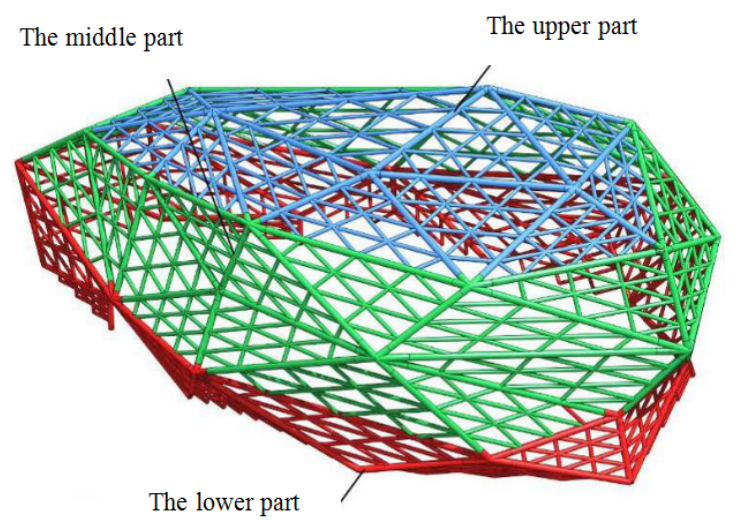

Fig. 6. Net shell zoning maps

(1) The results of linear buckling analysis showed that: the higher the buckling mode order, the greater the eigenvalue; the buckling deformation is mainly out of plane, and concentrated in the upper part of the structure, so the triangular mesh has large in-plane stiffness and small out-plane stiffness; the upper part of the structure is the prominent part of instability; with the increase of the order of buckling modes, the development of buckling deformation gradually transits from the upper part of the structure to the middle part of the structure, the deformation of the lower part is small;

(2) The results of nonlinear buckling analysis showed that: according to the reference [2], the critical load factor of the reticulated shell is 10.32; the upper part of the reticulated shell with no initial defect is the most sensitive part to the defect; the structure with initial defect will significantly reduce the stability bearing capacity of the shell; the larger the initial defect, the more unstable the structure is.

\section{Acknowledgments}

Natural Science Foundation of Inner Mongolia Autonomous Region(2017MS0517).

National Natural Science Foundation of China (51808357; 51638009)

\section{References}

1. Cai Jian, He Sheng, Jiang Zhengrong, et al. Investigation On Stability Analysis Method Of Single Layer Latticed Shells[J]. Engineering Mechanics, 2015, 32(7): 103-11

2. Ministry of Housing and Urban-Rural Development of the People's Republic of China. Technical specification for space frame structures: GB500102010[S]. Beijing: China Architecture \& Building Press, 2010

3. Li Jiajun. Analysis on Stability of the Single-layer Folded-plane Reticulated Shell Structure and Cast Steel Tubular Joint[D]. Shenyang: Shenyang Jianzhu University, 2013

4. MIDAS Information Technology Co., Ltd. MIDAS/Gen User Manual[M]. Beijing: 2019
5. Nie Guibo, Liu Kun, Zhi Xudong, et al. Performance-based seismic design of reticulated shells $[\mathrm{J}]$. China civil engineering journal, 2018, 51(1): $8-12$ 